\title{
Recuperação de solos afetados por sais pela aplicação de gesso de jazida e calcário no Nordeste do Brasil ${ }^{1}$
}

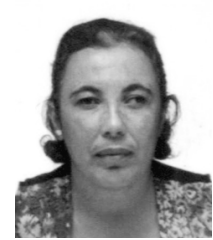

\author{
Maria de F. C. Barros ${ }^{2}$, Maurício P. F. Fontes ${ }^{3}$, Víctor H. Alvarez V. ${ }^{4}$ \& Hugo A. Ruiz ${ }^{5}$
}

\author{
1 Parte da Tese de Doutorado do primeiro autor apresentada à UFV/MG \\ 2 UFRPE/DEPA. CEP 52171-900, Recife, PE. Fone: (81) 3302-1220. E-mail: fatimacbarros@bol.com.br (Foto) \\ 3 DS/UFV. CEP 36571-000, Viçosa, MG. Fone: (31) 3899-1057. E-mail: mpfontes@mail.ufv.br \\ 4 DPS/UFV. Fone: (31) 3899-1061. E-mail: vhav@mail.ufv.br \\ 5 DPS/UFV. Fone: (31) 3899-1052. E-mail: hruiz@mail.com.br
}

Protocolo 48 - 17/3/2003 - Aprovado em 6/2/2004

Resumo: Objetivando-se avaliar o efeito da aplicação de corretivos químicos sobre as propriedades físicas e químicas, bem como na recuperação de solos afetados por sais do Perímetro Irrigado de Custódia, no Estado de Pernambuco, Brasil, realizou-se um experimento em colunas de solo, instaladas no Laboratório de Salinidade do Solo da Universidade Federal Rural de Pernambuco. Os tratamentos foram dispostos em um delineamento em blocos casualizados, com arranjamento fatorial de quatro solos, dois métodos de aplicação de gesso e gesso + calcário (aplicados na superfície e incorporados nos primeiros $5 \mathrm{~cm}$ da coluna de solo), duas combinações dos corretivos ( $100 \%$ de gesso $+0 \%$ de calcário e $80 \%$ de gesso $+20 \%$ de calcário), calculados com base na necessidade de gesso dos solos, e quatro faixas de granulometria de gesso $(2,0-1,0,1,0$ $0,5,0,5-0,3$ e $<0,3 \mathrm{~mm}$ ) com três repetições. No extrato da pasta saturada dos solos foram avaliados a condutividade elétrica (CE) e os cátions solúveis. A aplicação de gesso e da mistura de gesso + calcário revelou-se uma técnica eficaz de correção da sodicidade dos solos em estudo, indicada por um efeito positivo sobre as características físicas e químicas dos solos, podendo ser recomendadas com fontes de cálcio para recuperação de solos salino-sódicos. Entre as granulometrias de gesso, as mais finas, $(0,5-0,3 \mathrm{~mm}$ e $<0,3 \mathrm{~mm})$, apresentaram melhor desempenho na lixiviação dos sais e na conseqüente redução na relação de adsorsão de sódio (RAS) do extrato de saturação.

Palavras-chave: solos salino-sódicos, gesso, calcário, salinidade, RAS

\section{Reclamation of salt-affected soils in Northeast Brazil with application of mined gypsum and limestone}

\begin{abstract}
With the objective of evaluating the effect of the application of chemical amendments on the physical and chemical properties as well as on reclamation of the salt-affected soils in the "Perímetro Irrigado de Custódia", in Pernambuco state, Brazil, an experiment was carried out in soil columns installed at the Soil Salinity Laboratory of the Universidade Federal Rural de Pernambuco. The treatments were arranged in a randomized block design in a factorial scheme consisting of four soils, two methods of applications of gypsum and gypsum + limestone (applied on the surface and incorporated into the first $5 \mathrm{~cm}$ of the soil column), two combinations of the chemical amendments (100\% gypsum and $80 \%$ gypsum $+20 \%$ limestone), calculated on the basis of gypsum requirement of soils and four granulometry gypsum fractions (2.0 - 1.0; $1.0-0.5 ; 0.5-0.3$ and $<0.3 \mathrm{~mm}$ ) with three replications. In the saturation extract the electrical conductivity and the soluble cations were determined. The application of gypsum and the mixture of gypsum + limestone showed to be efficient for correction of soil sodicity, which was indicated by a positive effect on the physical and chemical characteristics of the soils, therefore these may be recommended as calcium source for the reclamation of the saline-sodic soils. Among the gypsum granulometry, the fine fractions $(0.5-0.3$ and $<0.3 \mathrm{~mm})$, presented better performance in leaching salt and in the reduction of sodium adsorption ratio (SAR) in saturation extract.
\end{abstract}

Key words: saline-sodic soils, gypsum, limestone, salinity, SAR 


\section{INTRODUÇÃO}

Normalmente, solos afetados por sais são encontrados em zonas áridas e semi-áridas, onde a evaporação é superior à precipitação. A drenagem interna deficiente apresentada em alguns solos dessas regiões, juntamente com a excessiva evaporação produz a acumulação de sais solúveis e o incremento do sódio trocável na superfície e/ou na subsuperfície dos solos.

A região de insuficiência hídrica do Nordeste brasileiro cobre uma superfície de 150 milhões de hectares. Nessa região a maioria dos perímetros de irrigação apresenta solos com alto teor de salinidade e/ou sódio trocável, que provocam desde a diminuição nos rendimentos das culturas até o abandono das áreas exploradas. Aproximadamente $20 \%$ da área total dos perímetros irrigados implantados no Estado de Pernambuco encontram-se com problemas de salinidade e/ou sodicidade (Magalhães, 1995).

A recuperação de solos afetados por sais tem como objetivo principal à redução da concentração dos sais solúveis e do sódio trocável no perfil do solo, a um nível não prejudicial ao desenvolvimento das culturas. A diminuição do grau de salinidade envolve o processo de solubilização e a conseqüente remoção pela água de percolação, enquanto a diminuição do teor de sódio trocável envolve o seu deslocamento do complexo de troca pelo cálcio antes do processo de lixiviação.

Por apresentar baixo custo e relativa abundância com que é encontrado em várias partes do mundo, o gesso é o corretivo mais utilizado para recuperação de solos sódicos e salinosódicos. Magalhães (1995) cita que Pernambuco possui 83\% das reservas de gesso de jazidas do País, localizadas próximas aos perímetros irrigados.

A eficiência do gesso como corretivo é dependente da dissolução. Alguns dos fatores que influenciam a taxa de dissolução do gesso no solo são a granulométrica das partículas do gesso e o método de aplicação do corretivo.

$\mathrm{Na}$ Austrália, a recomendação de calcário para a redução de problemas de sodicidade é pouco utilizada, em razão de sua baixa solubilidade em relação ao gesso (McKenzie et al. 1995); entretanto, a aplicação de calcário em solos sódicos e salinosódicos com $\mathrm{pH}<7,5$ vem sendo recomendada há várias décadas (Richards, 1954).

O presente trabalho teve como objetivos avaliar a influência do tamanho das partículas e do método de aplicação do gesso nas propriedades físicas, químicas e na recuperação de solos salino-sódicos e testar a aplicação de gesso + calcário como alternativa para a recuperação de solos afetados por sódio.

\section{MATERIAL E MÉTODOS}

O ensaio em coluna de solo foi instalado no Laboratório de Salinidade do Solo da Universidade Federal Rural Pernambuco
(UFRPE). Para este estudo, coletaram-se quatro amostras superficiais $(0-30 \mathrm{~cm})$ de solos salino-sódicos no Perímetro Irrigado de Custódia, no Estado de Pernambuco, Brasil.

Depois de coletadas, as amostras foram secas ao ar, destorroadas e passadas em peneira de $2 \mathrm{~mm}$ de malha.

A pasta saturada foi preparada seguindo-se a metodologia descrita por Richards (1954). No extrato da pasta saturada determinaram-se a condutividade elétrica (CE), o pH, o cálcio e o magnésio, por espectrofotometria de absorção atômica; o sódio e o potássio por fotometria de chama; o carbonato e o bicarbonato por titulação com $\mathrm{H}_{2} \mathrm{SO}_{4}$, e o cloreto por titulação com $\mathrm{AgNO}_{3}$ (EMBRAPA,1997). O sulfato foi determinado por colorimetria com precipitação com cromato de bário, seguindose a metodologia descrita por Magalhães (1987).

Utilizando-se os valores obtidos para cálcio, magnésio e sódio solúveis, quantificaram-se os valores para a relação de adsorção de sódio (RAS) usando-se a expressão:

$$
\mathrm{RAS}=\left(\mathrm{Na}^{+}\right) /\left[\left(\mathrm{Ca}^{2+}+\mathrm{Mg}^{2+}\right) / 2\right]^{1 / 2}
$$

Aplicando-se a metodologia sugerida pela EMBRAPA (1997), determinaram-se o pH dos solos na relação solo-água $(1: 2,5)$, a condutividade hidráulica pelo método descrito para solos com estrutura deformada e a composição granulométrica, após lavagem com etanol a $60 \%$, até a eliminação total de cloretos. As características químicas e físicas das amostras encontram-se na Tabela 1.

Os tratamentos foram dispostos em um delineamento em blocos casualizados, com arranjamento fatorial $4 \times 2 \times 2 \times 4$ (quatro solos, duas formas de aplicação, duas combinação de gesso e calcário e quatro granulometrias) com três repetições, totalizando 192 unidades experimentais.

A necessidade de gesso determinada pelo método de Schoonover M-1 (Barros \& Magalhães, 1989) foi aplicada ao solo pela combinação de gesso e calcário, de acordo com os seguintes tratamentos: $100 \%$ gesso $+0 \%$ calcário e $80 \%$ gesso $+20 \%$ calcário, através de dois métodos de aplicação; primeiro o gesso e o gesso + calcário foram aplicados uniformemente sobre a superfície de cada coluna de solo; na segunda metodologia, o gesso e o gesso + calcário foram incorporados aos primeiros $5 \mathrm{~cm}$ de solo, antes do acondicionamento na coluna.

As frações granulométricas do gesso utilizadas foram: partículas com diâmetro entre 2,0 e 1,0 mm (G1); partículas com diâmetro entre 1,0 e $0,5 \mathrm{~mm}(\mathrm{G} 2)$; partículas com diâmetro entre 0,5 e $0,3 \mathrm{~mm}$ (G3) e partículas com diâmetro $<0,3 \mathrm{~mm}(\mathrm{G} 4)$. A granulometria do calcário utilizada foi de partículas com diâmetro entre 2,0 e 1,0 mm.

As amostras de solo foram acondicionadas nas colunas, de modo a se aproximarem da densidade do solo de cada amostra,

Tabela 1. Características químicas e físicas dos solos

\begin{tabular}{|c|c|c|c|c|c|c|c|c|c|c|c|c|c|}
\hline \multirow{3}{*}{ Solo } & & Solú & eis no & Extra & da Past & Satur & ada & & \multirow{3}{*}{$\mathrm{pH}$} & \multirow{3}{*}{ 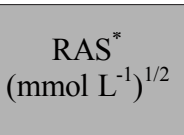 } & \multirow{3}{*}{$\begin{array}{l}\mathrm{CE}^{* *} \\
\mathrm{dS} \mathrm{m}{ }^{-1}\end{array}$} & \multirow{3}{*}{$\begin{array}{l}\text { Classe } \\
\text { Textural }\end{array}$} & \multirow{3}{*}{$\begin{array}{l}\text { Condutividade } \\
\text { Hidráulica } \\
\mathrm{cm} \mathrm{h}^{-1}\end{array}$} \\
\hline & $\mathrm{Na}^{+}$ & $\mathrm{Ca}^{2+}$ & $\mathrm{Mg}^{2+}$ & $\mathrm{K}^{+}$ & $\mathrm{Cl}^{-}$ & $\mathrm{SO}_{4}{ }^{2-}$ & $\mathrm{CO}_{3}{ }^{2-}$ & $\mathrm{HCO}_{3}^{-}$ & & & & & \\
\hline & \multicolumn{8}{|c|}{$\mathrm{mmol}_{\mathrm{c}} \mathrm{L}^{-1}$} & & & & & \\
\hline S1 & 55,60 & 3,62 & 2,53 & 0,63 & 60,06 & 1,02 & 0,00 & 0,80 & 7,01 & 31,7 & 6,12 & Franco & 0,17 \\
\hline $\mathrm{S} 2$ & 74,86 & 5,46 & 4,34 & 0,84 & 84,12 & 1,35 & 0,00 & 0, & 7,17 & & 8 , & Franco & \\
\hline $\mathrm{S} 3$ & 164,50 & 4,60 & 3,87 & 0,33 & 173,00 & 1,46 & 0,00 & 1,25 & 7,30 & 79,94 & 17,20 & Franco argilo arenoso & 0,00 \\
\hline S4 & 50,07 & 3,28 & 2,75 & 0,51 & 53,48 & 0,83 & 0,00 & 0,30 & 6,90 & 28,84 & 5,24 & Franco arenoso & 0,29 \\
\hline
\end{tabular}

"Relação de adsorção de sódio

** Condutividade elétrica do extrato da pasta saturada 
em tubos plásticos de PVC com $10 \mathrm{~cm}$ de diâmetro e $17 \mathrm{~cm}$ de altura, divididas em duas camadas, cada uma com $5 \mathrm{~cm}$ de espessura, tendo em sua base um sistema de drenagem; em seguida, as colunas de solo foram lixiviadas com água destilada, mantendo-se um nível constante de $2 \mathrm{~cm}$ acima da superfície do solo. Para lixiviação utilizou-se uma lâmina de água equivalente a duas vezes o volume de poros, tendo sido aplicado $600 \mathrm{~mL}$.

Depois da lixiviação, as colunas de solo foram seccionadas no intervalo de $5 \mathrm{~cm}$ e o solo de cada camada foi seco ao ar, destorroado e passado em peneira de $2 \mathrm{~mm}$ de abertura determinando-se, em seguida, a condutividade elétrica (CE) e os cátions solúveis do extrato da pasta saturada. Quantificaramse também os valores para relação de adsorção de sódio (RAS) de acordo com a metodologia descrita anteriormente.

Os dados obtidos foram interpretados por meio de análise de variância e de regressão, testando-se diversos modelos. Os critérios para escolha do modelo foram o maior valor do coeficiente de determinação ajustado e a significância dos coeficientes da equação de regressão.

\section{RESULTADOS E DISCUSSÃO}

A aplicação dos corretivos e a lixiviação dos sais resultaram em uma grande variação nos valores de cálcio, magnésio, sódio e potássio solúveis e na condutividade elétrica do extrato de saturação, em relação aos valores encontrados antes da aplicação dos tratamentos (Tabela 2). A concentração de cálcio aumentou com o decréscimo na granulometria das partículas de gesso devido, provavelmente, à maior solubilização do gesso; portanto, houve maior concentração de cálcio em solução, quando foram utilizadas partículas de menor diâmetro, enquanto, os valores de sódio solúveis mostraram o oposto, sendo os maiores valores registrados para os tratamentos de gesso de maior granulometria, comportamento observado tanto para a camada de $0-5 \mathrm{~cm}$, como para a de $5-10 \mathrm{~cm}$.

Pode-se constatar também que tanto a concentração de sódio como a RAS aumentaram em profundidade, o que se deve, provavelmente, ao rápido decréscimo da concentração de cálcio em solução, em virtude das reações de troca ocorridas

Tabela 2. Composição do extrato de saturação do solo para diferentes amostras

\begin{tabular}{|c|c|c|c|c|c|c|c|c|c|c|c|c|c|c|}
\hline \multirow{4}{*}{$\begin{array}{c}\text { Forma } \\
\text { de } \\
\text { Aplicação }\end{array}$} & \multirow{4}{*}{ Mistura } & \multirow{4}{*}{${ }^{\circ 0 \circ} \mathrm{G}$} & \multicolumn{8}{|c|}{ Cátions Solúveis $\left(\mathrm{mmol}_{\mathrm{c}} \mathrm{L}^{-1}\right)$} & \multirow{2}{*}{\multicolumn{2}{|c|}{$\begin{array}{c}{ }^{\#} \mathrm{CE} \\
\left(\mathrm{dS} \mathrm{m}{ }^{-1}\right)\end{array}$}} & \multirow{2}{*}{\multicolumn{2}{|c|}{$\begin{array}{c}{ }^{\#} \mathrm{RAS} \\
\left(\left(\mathrm{mmol}^{-1} \mathrm{~L}^{-1}\right)^{1 / 2}\right)\end{array}$}} \\
\hline & & & \multicolumn{2}{|c|}{$\mathrm{Ca}^{2+}$} & \multicolumn{2}{|c|}{$\mathrm{Mg}^{2+}$} & \multicolumn{2}{|c|}{$\mathrm{Na}^{+}$} & \multicolumn{2}{|c|}{$\mathrm{K}^{+}$} & & & & \\
\hline & & & \multicolumn{12}{|c|}{ Profundidade $-\mathrm{cm}$} \\
\hline & & & $0-5$ & $5-10$ & $0-5$ & $5-10$ & $0-5$ & $5-10$ & $0-5$ & $5-10$ & $0-5$ & $5-10$ & $0-5$ & $5-10$ \\
\hline \multicolumn{15}{|l|}{ A. Amostras $S_{1}$} \\
\hline \multirow{8}{*}{$\begin{array}{l}\text { Sup. } \\
{ }^{*} \mathrm{~B} 1\end{array}$} & \multirow{4}{*}{${ }^{\circ} \mathrm{C} 1$} & G1 & 8,20 & 3,01 & 0,28 & 0,66 & 4,70 & 6,38 & 0,13 & 0,08 & 1,36 & 0,99 & 2,29 & 4,72 \\
\hline & & $\mathrm{G} 2$ & 9,25 & 4,21 & 0,28 & 0,67 & 4,08 & 5,53 & 0,14 & 0,09 & 1,44 & 1,05 & 1,87 & 3,54 \\
\hline & & G3 & 11,18 & 6,26 & 0,27 & 0,68 & 3,57 & 5,16 & 0,13 & 0,08 & 1,58 & 1,26 & 1,50 & 2,77 \\
\hline & & G4 & 12,63 & 8,90 & 0,28 & 0,69 & 3,01 & 4,37 & 0,14 & 0,09 & 1,66 & 1,45 & 1,19 & 2,00 \\
\hline & \multirow{4}{*}{${ }^{\circ} \mathrm{C} 2$} & G1 & 7,02 & 2,06 & 0,28 & 0,67 & 5,00 & 6,73 & 0,14 & 0,08 & 1,23 & 0,88 & 2,63 & 5,85 \\
\hline & & G2 & 8,00 & 3,53 & 0,29 & 0,69 & 4,19 & 5,95 & 0,15 & 0,08 & 1,28 & 0,99 & 2,07 & 4,10 \\
\hline & & G3 & 10,37 & 5,22 & 0,28 & 0,67 & 3,91 & 5,57 & 0,14 & 0,08 & 1,46 & 1,17 & 1,70 & 3,24 \\
\hline & & G4 & 11,95 & 7,87 & 0,27 & 0,68 & 3,33 & 4,80 & 0,14 & 0,08 & 1,55 & 1,32 & 1,35 & 2,32 \\
\hline \multirow{8}{*}{$\begin{array}{l}\text { Inc. } \\
* * \mathrm{~B} 2\end{array}$} & \multirow{4}{*}{${ }^{\circ} \mathrm{C} 1$} & G1 & 9,97 & 4,69 & 0,27 & 0,68 & 4,02 & 5,86 & 0,15 & 0,08 & 1,51 & 1,10 & 1,78 & 3,58 \\
\hline & & G2 & 11,32 & 5,92 & 0,27 & 0,70 & 3,44 & 5,03 & 0,14 & 0,08 & 1,58 & 1,15 & 1,43 & 2,77 \\
\hline & & G3 & 13,01 & 7,67 & 0,29 & 0,69 & 3,07 & 4,67 & 0,14 & 0,09 & 1,77 & 1,33 & 1,19 & 2,28 \\
\hline & & G4 & 14,48 & 10,21 & 0,28 & 0,68 & 2,50 & 3,77 & 0,13 & 0,10 & 1,85 & 1,56 & 0,92 & 1,62 \\
\hline & \multirow{4}{*}{${ }^{\circ} \mathrm{C} 2$} & G1 & 8,85 & 3,70 & 0,29 & 0,69 & 4,50 & 6,26 & 0,13 & 0,08 & 1,42 & 1,04 & 2,11 & 4,24 \\
\hline & & G2 & 10,03 & 4,60 & 0,26 & 0,69 & 3,87 & 5,42 & 0,13 & 0,08 & 1,47 & 1,09 & 1,71 & 3,35 \\
\hline & & G3 & 11,87 & 6,80 & 0,27 & 0,68 & 3,49 & 5,06 & 0,15 & 0,08 & 1,65 & 1,27 & 1,42 & 2,62 \\
\hline & & G4 & 13,33 & 9,30 & 0,27 & 0,68 & 2,93 & 4,23 & 0,13 & 0,08 & 1,76 & 1,42 & 1,12 & 1,89 \\
\hline \multicolumn{15}{|l|}{ B. Amostra $\mathrm{S}_{2}$} \\
\hline \multirow{8}{*}{$\begin{array}{l}\text { Sup. } \\
\text { *B1 }\end{array}$} & \multirow{4}{*}{${ }^{\circ} \mathrm{C} 1$} & G1 & 12,12 & 3,63 & 0,52 & 0,96 & 6,22 & 8,60 & 0,22 & 0,16 & 1,80 & 1,35 & 2,48 & 5,70 \\
\hline & & G2 & 15,88 & 5,24 & 0,51 & 0,96 & 5,40 & 7,74 & 0,21 & 0,15 & 2,10 & 1,52 & 1,89 & 4,40 \\
\hline & & G3 & 17,95 & 8,30 & 0,52 & 0,96 & 4,85 & 6,92 & 0,20 & 0,14 & 2,25 & 1,66 & 1,60 & 3,22 \\
\hline & & G4 & 19,87 & 9,99 & 0,51 & 0,96 & 4,12 & 5,96 & 0,21 & 0,15 & 2,36 & 1,75 & 1,29 & 2,55 \\
\hline & \multirow{4}{*}{${ }^{\circ} \mathrm{C} 2$} & G1 & 11,24 & 2,55 & 0,52 & 0,95 & 6,52 & 8,99 & 0,22 & 0,17 & 1,72 & 1,25 & 2,70 & 6,83 \\
\hline & & G2 & 14,81 & 4,09 & 0,51 & 0,96 & 5,82 & 8,12 & 0,21 & 0,16 & 1,96 & 1,39 & 2,10 & 5,14 \\
\hline & & G3 & 16,83 & 7,19 & 0,51 & 0,96 & 5,13 & 7,35 & 0,20 & 0,15 & 2,15 & 1,60 & 1,74 & 3,65 \\
\hline & & G4 & 18,76 & 8,81 & 0,50 & 0,98 & 4,39 & 6,30 & 0,20 & 0,16 & 2,27 & 1,68 & 1,42 & 2,85 \\
\hline \multirow{8}{*}{$\begin{array}{l}\text { Inc. } \\
* * \text { B2 }\end{array}$} & & G1 & 5,98 & 0,53 & 0,95 & 5,58 & 7,83 & 0,24 & 0,16 & 2,04 & 1,48 & 2,04 & 4,21 & 5,98 \\
\hline & & G2 & 7,90 & 0,54 & 0,97 & 5,05 & 7,02 & 0,20 & 0,16 & 2,27 & 1,64 & 1,70 & 3,33 & 7,90 \\
\hline & $\mathrm{Cl}$ & G3 & 11,02 & 0,52 & 0,96 & 4,25 & 6,44 & 0,20 & 0,17 & 2,44 & 1,85 & 1,34 & 2,63 & 11,02 \\
\hline & & G4 & 12,55 & 0,51 & 0,98 & 3,62 & 5,20 & 0,21 & 0,16 & 2,53 & 1,94 & 1,09 & 2,00 & 12,55 \\
\hline & & G1 & 13,08 & 4,88 & 0,52 & 0,96 & 5,94 & 8,45 & 0,22 & 0,16 & 1,90 & 1,41 & 2,28 & 4,97 \\
\hline & ${ }^{\circ}{ }^{\circ} \mathrm{C}$ & G2 & 16,47 & 6,91 & 0,53 & 0,97 & 5,35 & 7,43 & 0,20 & 0,15 & 2,19 & 1,56 & 1,84 & 3,74 \\
\hline & $c 2$ & G3 & 18,52 & 9,74 & 0,52 & 0,98 & 4,62 & 6,68 & 0,21 & 0,17 & 2,33 & 1,72 & 1,50 & 2,89 \\
\hline & & G4 & 20,68 & 11,32 & 0,52 & 0,97 & 3,98 & 5,65 & 0,22 & 0,16 & 2,45 & 1,80 & 1,22 & 2,28 \\
\hline
\end{tabular}


Continuação da Tabela 2

\begin{tabular}{|c|c|c|c|c|c|c|c|c|c|c|c|c|c|c|}
\hline \multirow{4}{*}{$\begin{array}{c}\text { Forma } \\
\text { de } \\
\text { Aplicação }\end{array}$} & \multirow{4}{*}{ Mistura } & \multirow{4}{*}{${ }^{\circ 0 \circ} \mathrm{G}$} & \multicolumn{8}{|c|}{ Cátions Solúveis $\left(\mathrm{mmol}_{\mathrm{c}} \mathrm{L}^{-1}\right)$} & \multirow{2}{*}{\multicolumn{2}{|c|}{$\begin{array}{c}{ }^{\#} \mathrm{CE} \\
\left(\mathrm{dS} \mathrm{m}{ }^{-1}\right)\end{array}$}} & \multirow{2}{*}{\multicolumn{2}{|c|}{$\begin{array}{c}{ }^{\# \#} \mathrm{RAS}^{-1} \\
\left(\left(\mathrm{mmol} \mathrm{L}^{-1}\right)^{1 / 2}\right)\end{array}$}} \\
\hline & & & \multicolumn{2}{|c|}{$\mathrm{Ca}^{2+}$} & \multicolumn{2}{|c|}{$\mathrm{Mg}^{2+}$} & \multicolumn{2}{|c|}{$\mathrm{Na}^{+}$} & \multicolumn{2}{|c|}{$\mathrm{K}^{+}$} & & & & \\
\hline & & & \multicolumn{12}{|c|}{ Profundidade $-\mathrm{cm}$} \\
\hline & & & $0-5$ & $5-10$ & $0-5$ & $5-10$ & $0-5$ & $5-10$ & $0-5$ & $5-10$ & $0-5$ & $5-10$ & $0-5$ & $5-10$ \\
\hline \multicolumn{15}{|l|}{ C. Amostra $\mathrm{S}_{3}$} \\
\hline \multirow{6}{*}{$\begin{array}{l}\text { Sup. } \\
\text { *B1 }\end{array}$} & \multirow{2}{*}{${ }^{\circ} \mathrm{C} 1$} & G1 & 19,94 & 10,34 & 0,18 & 0,31 & 11,18 & 17,12 & 0,05 & 0,06 & 3,02 & 2,75 & 3,53 & 7,42 \\
\hline & & G2 & 21,92 & 13,17 & 0,18 & 0,33 & 9,80 & 15,16 & 0,07 & 0,05 & 3,12 & 2,83 & 2,95 & 5,84 \\
\hline & \multirow{4}{*}{${ }^{\circ} \mathrm{C} 2$} & G1 & 18,77 & 9,15 & 0,18 & 0,35 & 11,62 & 17,54 & 0,07 & 0,06 & 2,95 & 2,66 & 3,78 & 8,05 \\
\hline & & G2 & 20,80 & 12,01 & 0,16 & 0,31 & 9,94 & 15,82 & 0,07 & 0,06 & 3,01 & 2,76 & 3,07 & 6,38 \\
\hline & & G3 & 23,01 & 14,97 & 0,17 & 0,32 & 8,21 & 12,99 & 0,08 & 0,06 & 3,14 & 2,85 & 2,41 & 4,70 \\
\hline & & G4 & 25,44 & 17,92 & 0,18 & 0,33 & 6,57 & 10,51 & 0,09 & 0,07 & 3,24 & 2,93 & 1,84 & 3,48 \\
\hline \multirow{5}{*}{$\begin{array}{l}\text { Inc. } \\
* * \mathrm{~B} 2\end{array}$} & \multirow{2}{*}{${ }^{\circ} \mathrm{C} 1$} & G1 & 22,00 & 12,87 & 0,18 & 0,34 & 10,53 & 16,41 & 0,09 & 0,07 & 3,20 & 2,90 & 3,16 & 6,39 \\
\hline & & G2 & 23,88 & 15,70 & 0,18 & 0,33 & 9,38 & 14,43 & 0,07 & 0,06 & 3,26 & 2,99 & 2,70 & 5,10 \\
\hline & \multirow{3}{*}{${ }^{\circ} \mathrm{C} 2$} & G2 & 22,77 & 14,93 & 0,17 & 0,33 & 9,66 & 14,87 & 0,09 & 0,07 & 3,17 & 2,91 & 2,85 & 5,39 \\
\hline & & G3 & 25,25 & 17,40 & 0,17 & 0,32 & 7,60 & 12,58 & 0,08 & 0,06 & 3,30 & 3,00 & 2,13 & 4,23 \\
\hline & & G4 & 27,36 & 20,93 & 0,18 & 0,33 & 6,03 & 9,74 & 0,07 & 0,07 & 3,42 & 3,10 & 1,63 & 2,99 \\
\hline \multicolumn{15}{|l|}{ D. Amostra $\mathrm{S}_{4}$} \\
\hline \multirow{7}{*}{$\begin{array}{l}\text { Sup. } \\
*_{B} 1\end{array}$} & \multirow{4}{*}{${ }^{\circ} \mathrm{C} 1$} & G1 & 6,91 & 2,78 & 0,24 & 0,47 & 3,99 & 5,54 & 0,11 & 0,04 & 1,20 & 0,80 & 2,13 & 4,39 \\
\hline & & $\mathrm{G} 2$ & 9,00 & 4,05 & 0,24 & 0,47 & 3,66 & 4,74 & 0,11 & 0,05 & 1,33 & 0,85 & 1,70 & 3,17 \\
\hline & & G3 & 10,95 & 5,28 & 0,24 & 0,49 & 3,33 & 3,96 & 0,12 & 0,06 & 1,49 & 0,96 & 1,41 & 2,35 \\
\hline & & G4 & 12,41 & 6,65 & 0,23 & 0,48 & 2,36 & 2,84 & 0,11 & 0,07 & 1,54 & 1,04 & 0,94 & 1,50 \\
\hline & \multirow{3}{*}{${ }^{\circ} \mathrm{C} 2$} & G1 & 5,72 & 1,95 & 0,22 & 0,44 & 4,37 & 6,10 & 0,11 & 0,06 & 1,07 & 0,72 & 2,55 & 5,62 \\
\hline & & $\mathrm{G} 2$ & 7,85 & 3,09 & 0,23 & 0,48 & 3,97 & 5,17 & 0,10 & 0,06 & 1,23 & 0,77 & 1,98 & 3,88 \\
\hline & & G3 & 9,91 & 4,35 & 0,23 & 0,48 & 3,68 & 4,40 & 0,11 & 0,06 & 1,40 & 0,89 & 1,64 & 2,83 \\
\hline $\begin{array}{l}\text { Inc. } \\
* * \mathrm{~B} 2\end{array}$ & & G4 & 13,41 & 7,23 & 0,25 & 0,47 & 2,25 & 2,90 & 0,10 & 0,06 & 1,58 & 1,10 & 0,86 & 1,48 \\
\hline
\end{tabular}

$(2-1 \mathrm{~mm}), \mathrm{G} 2(1-0,5 \mathrm{~mm}), \mathrm{G} 3(0,5-0,3 \mathrm{~mm})$ e G4 $(<0,3 \mathrm{~mm}) ;{ }^{*} \mathrm{CE}=$ Condutividade elétrica; e ${ }^{\# \# A S}=$ Relação de adsorção de sódio

na camada de $0-5 \mathrm{~cm}$. Durante o processo de correção de solos salino-sódicos, a solubilidade do gesso é aumentada pela redução dos coeficientes de atividade do cálcio e sulfato em solução com o tempo, o aumento da força iônica da solução tende a reduzir a intensidade das substituições do sódio pelo cálcio (Oster \& Frenkel, 1980; Oster, 1982; Sumner, 1995).

As aplicações de gesso e a combinação de gesso + calcário contribuíram para diminuição da sodicidade dos solos. As modificações verificadas na concentração de sais, expressa pela CE do extrato da pasta saturada após a aplicação dos corretivos (Figuras 1 e 2) comprovam a eficiência dos tratamentos utilizados na remoção dos sais solúveis e do sódio trocável do solo, justificada pela floculação das argilas decorrente da substituição do sódio adsorvido no complexo de troca pelo cálcio. Tal fato faz aumentar a permeabilidade dos solos, resultando em rápida infiltração e redistribuição da água no solo favorecendo, assim, a lixiviação dos sais. Segundo Gheyi et al. (1995), o gesso interfere na salinidade do solo aumentando-a, inicialmente, pela liberação de eletrólitos, e diminuindo-a no decorrer do tempo, pelo aumento da permeabilidade, que favorecem a lixiviação dos sais.

Os dados obtidos neste estudo estão de acordo com os observados por Suhayda et al. (1997) em experimento de campo, depois da aplicação de gesso incorporado à profundidade de $15 \mathrm{~cm}$. Os autores observaram significativo efeito em todas as propriedades do solo em relação ao tratamento-controle; os níveis de cloreto e de sódio solúveis e da condutividade elétrica, decresceram em 90, 43 e $32 \%$, respectivamente. Resultados similares sobre o efeito da aplicação de gesso no decréscimo da salinidade do solo foram também relatados por Rahman et al. (1996); esses autores observaram que a aplicação de gesso em solo salino-sódico aumentou a condutividade hidráulica do solo, favorecendo a lixiviação dos sais, o que resultou em redução na condutividade elétrica para valores $<4 \mathrm{dS} \mathrm{m}^{-1}$, tanto para a camada de 0-30 $\mathrm{cm}$ como para a de $30-60 \mathrm{~cm}$ de profundidade das colunas de solo.

No presente estudo observa-se que os dados apresentados para a RAS (Figuras 3 e 4), atingiram valores muito baixos, 

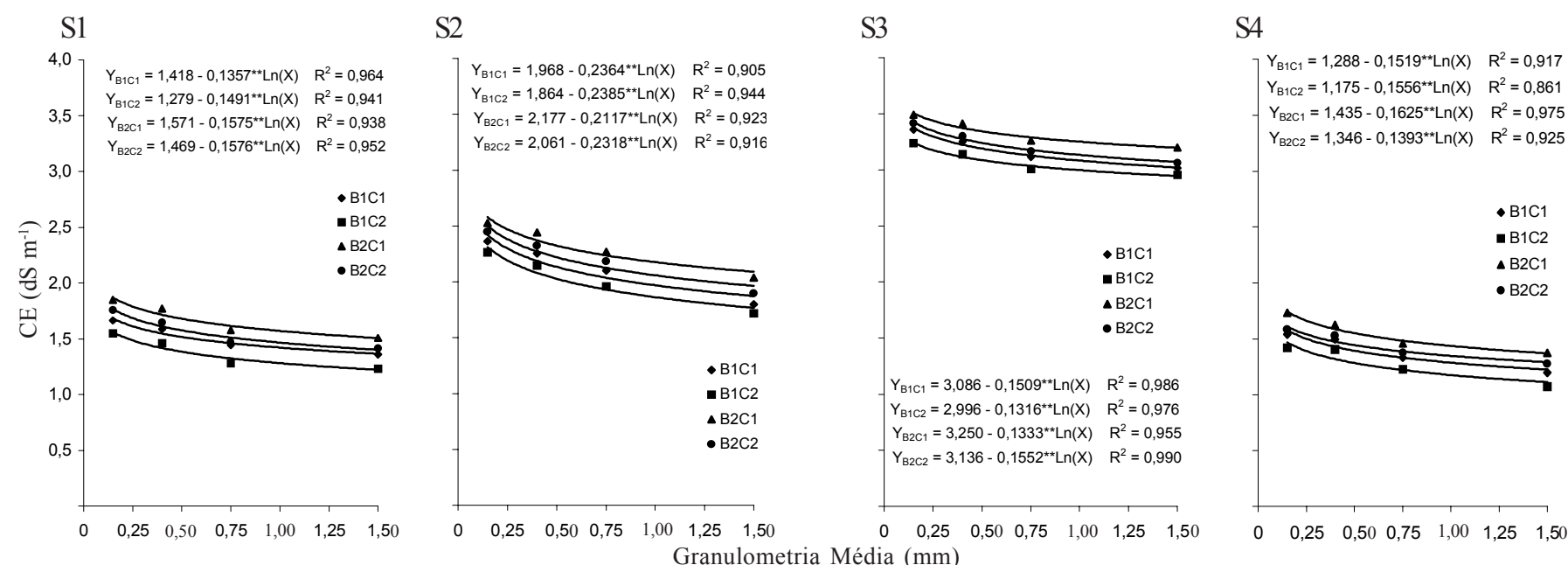

Figura 1. Condutividade elétrica (CE) do extrato de saturação na profundidade de $0-5 \mathrm{~cm}$ do solo, em relação à forma de aplicação dos corretivos na superfície (B1), incorporado (B2), mistura $\mathrm{Cl}$ (100\% de gesso ) e mistura $\mathrm{C} 2(80 \%$ de gesso $+20 \%$ de calcário) em função da granulometria média do gesso(G), G1 (1,5 mm), G2 (0,75 mm), G3 (0,4 mm) e G4 (0,15 mm), para 4 amostras de solos (S1, S2, S3 e S4)
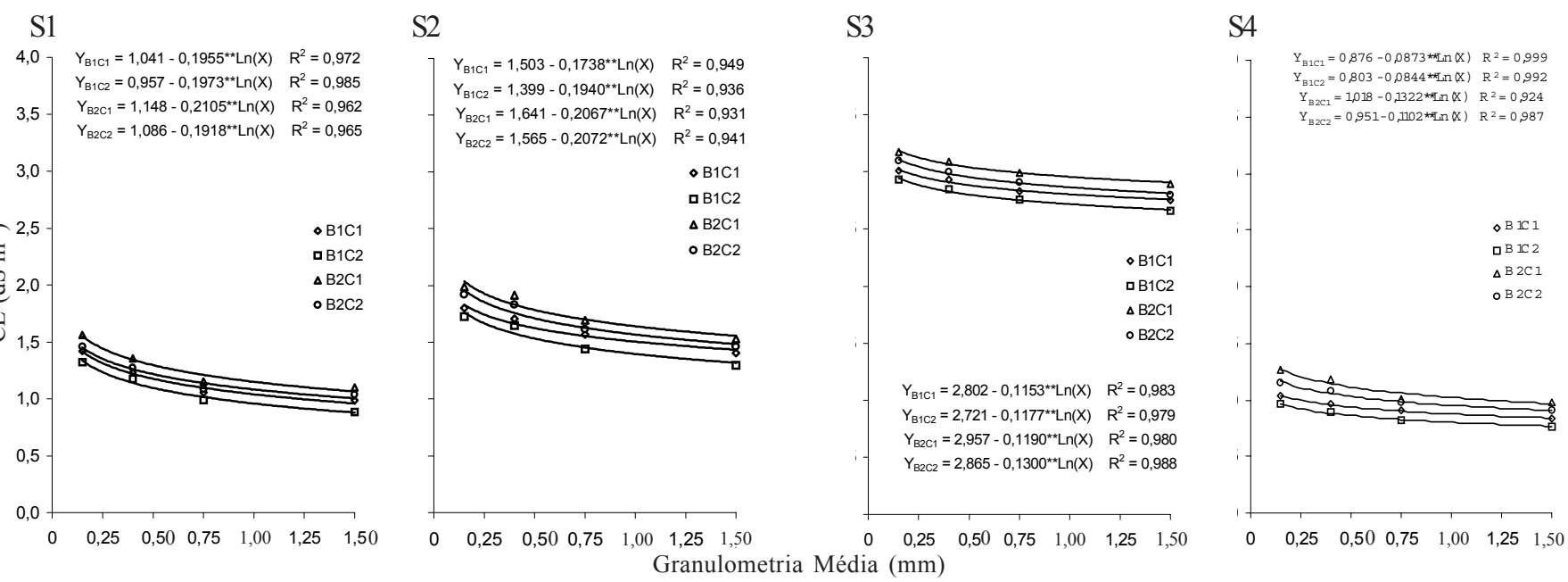

Figura 2. Condutividade elétrica (CE) do extrato de saturação na profundidade de $5-10 \mathrm{~cm}$ do solo, em relação à forma de aplicação dos corretivos na superfície (B1), incorporado (B2), mistura C1 (100\% de gesso) e mistura C2 ( $80 \%$ de gesso $+20 \%$ de calcário) em função da granulometria média do gesso (G), G1 (1,5 mm), G2 (0,75 mm), G3 (0,4 mm)e G4 (0,15 mm), para 4 amostras de solos (S1, S2, S3 e S4)

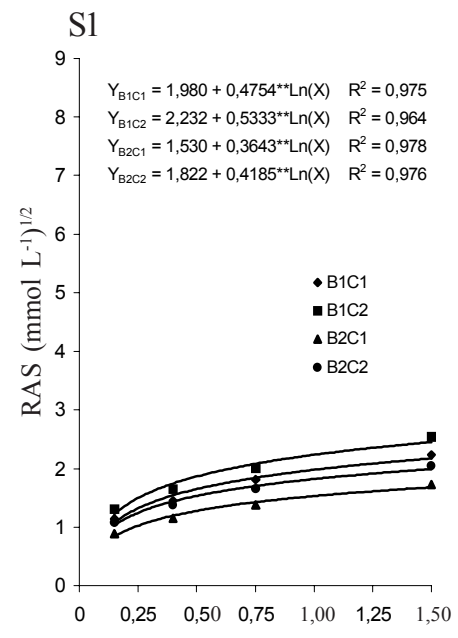

$\mathrm{S} 2$

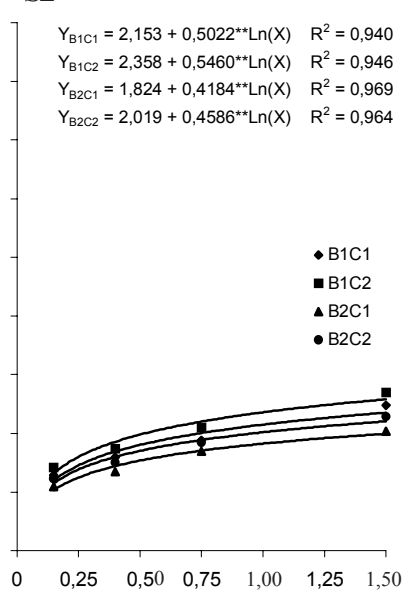

S3

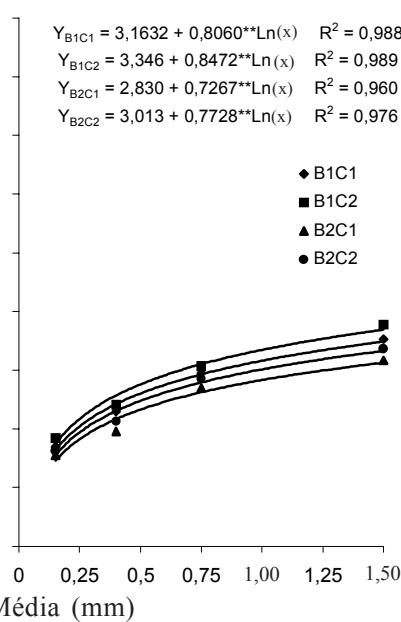

S4

$Y_{B 1 C 1}=1,828+0,4985^{* *} \operatorname{Ln}(X) \quad R^{2}=0,996$ $Y_{B 1 C 2}=2,163+0,5937^{* *} \operatorname{Ln}(X) \quad R^{2}=0,987$ $Y_{\mathrm{B} 2 \mathrm{C} 1}=1,388+0,3794^{* *} \operatorname{Ln}(X) \quad R^{2}=0,947$ $Y_{\mathrm{B} 2 \mathrm{C} 2}=1,619+0,4625^{* *} \operatorname{Ln}(X) \quad R^{2}=0,941$

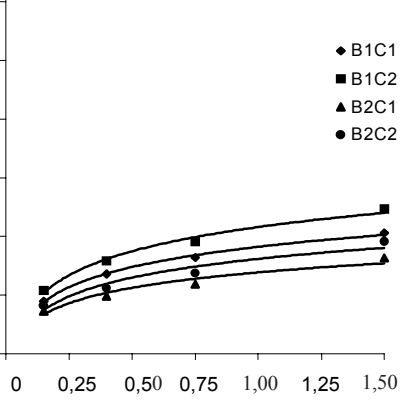

Figura 3. Relação de adsorção de sódio (RAS) do extrato de saturação na profundidade de 0-5 cm do solo, em relação à forma de aplicação dos corretivos na superfície (B1), incorporado (B2), mistura $\mathrm{C} 1$ (100\% de gesso) e mistura $\mathrm{C} 2$ ( $80 \%$ de gesso $+20 \%$ de calcário) em função da granulometria média do gesso $(\mathrm{G}), \mathrm{G} 1(1,5 \mathrm{~mm}), \mathrm{G} 2(0,75 \mathrm{~mm}), \mathrm{G} 3(0,4 \mathrm{~mm})$ e G4 $(0,15 \mathrm{~mm})$, para 4 amostras de solos (S1, S2, S3 e S4) 

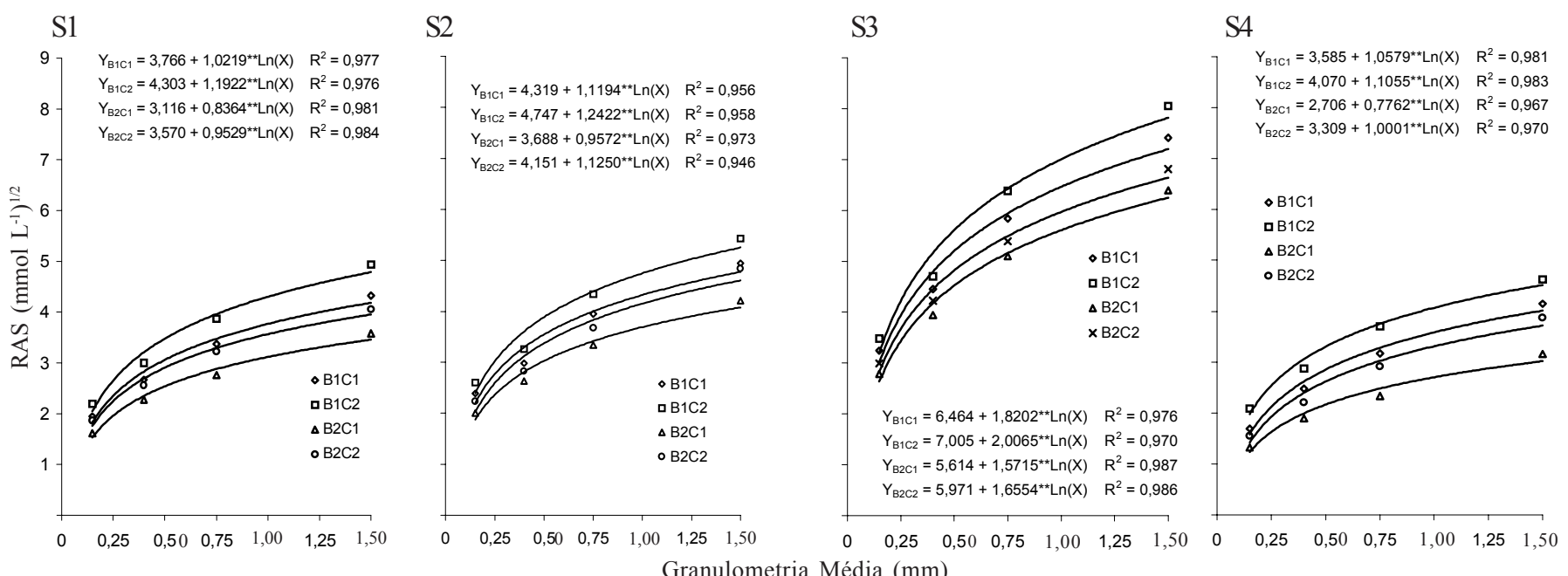

Figura 4. Relação de adsorção de sódio (RAS) do extrato de saturação na profundidade de 5-10 cm do solo, em relação à forma de aplicação dos corretivos na superfície (B1), incorporado (B2), mistura $\mathrm{C} 1$ (100\% de gesso) e mistura $\mathrm{C} 2$ ( $80 \%$ de gesso $+20 \%$ de calcário) em função da granulometria média do gesso, G1 (1,5 mm), G2 (0,75 mm), G3 (0,4 mm) e G4 (0,15 mm), para4 amostras de solos ( S1, S2, S3 eS4)

indicando que as aplicações de gesso e da combinação de gesso + calcário contribuíram para diminuição da sodicidade dos solos, semelhante ao ocorrido nos estudos de recuperação de solos sódicos em colunas de solo realizados por De Jong (1982). Adicionando 14,5; 29 e $58 \mathrm{mmol}$ de cálcio por coluna de solo, o autor constatou que a reação de troca entre $\mathrm{Ca}^{2+}-\mathrm{Na}^{+}$foi completa em amostra de solo que apresentava uma RAS de 100, e uma CE de $40 \mathrm{dS} \mathrm{m}^{-1}$. Pode-se observar, também, que todas as amostras de solos apresentaram a mesma tendência de maior diminuição da RAS com o aumento da superfície específica das partículas de gesso e este efeito foi mais pronunciado nos tratamentos que receberam os corretivos incorporados nos primeiros cinco centímetros da coluna de solo.

\section{CONCLUSÕES}

1. A aplicação de gesso e da mistura de gesso + calcário revelou-se técnica eficaz de correção da sodicidade dos solos em estudo, indicada por um efeito positivo sobre as características dos solos, podendo ser recomendadas como fontes de cálcio para recuperação de solos salino-sódicos.

2. O gesso, quando aplicado nas granulometrias mais finas $(0,5-0,3$ e $<0,3 \mathrm{~mm})$ mostrou melhor desempenho na redução da RAS do extrato de saturação dos solos, e na conseqüente substituição do sódio trocável .

\section{LITERATURA CITADA}

Barros, M.F.C.; Magalhães, A.F. Avaliações de métodos de determinação da necessidade de gesso em solos salinosódicos. Revista Brasileira de Ciência Solo, Campinas, v.13, p.119-123, 1989.

De Jong, E. Reclamation of solis contaminated by sodium chloride. Canadian Journal Soil Science, Quebec, v.62, p.351364, 1982.

EMBRAPA - Empresa Brasileira de Pesquisa Agropecuária. Manual de Métodos de análises de solo. 2 ed. Rio de Janeiro, 1997.212p.
Gheyi, H.R.; Azevedo, N.C.; Batista, M.A.F.; Santos, J.G.R. Comparação de métodos na recuperação de solo salinosódico. Revista Brasileira de Ciência Solo, Campinas, v.19, p.173-178, 1995 .

Magalhães, A.F. Métodos de análise químicas para solos salinos e sódicos. Recife: Departamento de Agronomia, UFRPE, 1987.30p.

Magalhães, A.F. Manejo da fertilidade dos solos irrigados: produtividade, degradação e correção. In: Reunião Brasileira de Fertilidade do Solo e Nutrição de Plantas, 21, 1995, Petrolina, PE. Anais... Petrolina, SBCS, 1995. p.77-86.

McKenzie, D.C.; Abbott, T.S.; Chan, K.Y.; Slavich, P.G.; Hall. D.J.M. The nature, distribution and management of sodic soils in New South Wales. In: Naidu, R.; Sumner, M.E.; Rengasamy, P. (eds.) Australian sodic soils: Distribution, properties and management. Victoria: CSRIO, 1995. p.246-264.

Oster, J.D. Gypsum usage in irrigated agriculture: A review. Fertilizer Research, Dordrecht, v.3, p.73-89, 1982.

Oster, J.D.; Frenkel, H. The chemistry of the reclamation of sodic soils with gypsum and lime. Soil Science Society of America Journal, Madison, v.44, p.41-15, 1980.

Rahman, A.H.A.; Dahab, M.H.; Mustafa, M.A. Impact of soil amendments on intermittent evaporation, moisture distribution and salt redistribution in saline-sodic clay soil columns. Soil Science, Baltimore, v.161, p.793-802, 1996.

Richards, L.A. Diagnosis and improvement of saline and alkali soils. Washington: US Department of Agriculture, 1954. 160p. USDA Agricultural Handbook, 60

Suhayda, C.G.; Yin, L.; Redmann, R.E.; Li, J. Gypsum amendment improves native grass establishment on saline-alkali solis in north-east China. Soil Use and Management, Oxford, v.13, p.43-47, 1997.

Sumner, M.E. Sodic soils: new perspectives. In: Naidu, R.; Sumner, M.E.; Regasamy, P. (eds.) Australian sodic soils: Distribution, properties and management. Victoria: CSIRO, 1995. p.1-34. 